\title{
Abordaje de la disfunción física en la formación inicial de profesionales de kinesiología/fisioterapia
}

\author{
Approach of physical dysfunction in the initial training of physiotherapists/physiotherapists
}

Abordagem da disfunção física na educação inicial de profissionais em cinesiología/fisioterapia

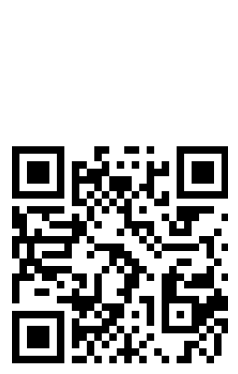

\author{
Máximo Escobar-Cabello \\ Universidad Católica del Maule \\ Talca, Chile \\ mescobar@ucm.cl \\ iD https://orcid.org/0000-0001-5780-207X \\ Héctor Cárcamo-Vásquez \\ Universidad del Bío-Bío \\ Chillán, Chile \\ hcarcamo@ubiobio.cl \\ https://orcid.org/0000-0003-2045-343X
}

Recibido • Received • Recebido: 17 / 01 / 2018

Corregido • Revised • Revisado: 03 / 06 / 2019

Aceptado • Accepted • Aprovado: $13 / 11 / 2019$

\begin{abstract}
Resumen: El ensayo identifica los factores estructurales del poder gubernamental que enfrentan las personas con disfunción física (PDF). Se integra al análisis la formación tradicional que reciben profesionales de la kinesiología/fisioterapia. El propósito de este abordaje es evidenciar que los subsidios de las estructuras sistémicas están capturados por una lógica de reproducción que nace desde la misma Organización Mundial de la Salud (OMS) y llegan a establecer, contradictoriamente, ante la complejidad que representa esta realidad, una conducta subsidiaria que responsabiliza a las propias PDF. Frente a estos acontecimientos, se recurre al enfoque sociocrítico para develar que las condicionantes estructurales del poder solo pueden administrar los insuficientes subsidios existentes. Y que a reglón seguido esta realidad debe transformarse en un núcleo problemático proritario para que sea incorporado en los procesos formativos de las matrices curriculares de los grupos kinesiólogos/ fisioterapeutas que interactúan con propiedad y frecuentemente en estos contextos. El documento muestra el escenario sociodemográfico y la descripción del status quo a manera de interpretar que, con la teoría de reproducción social o con los procesos de innovación curricular oficial, no se logra una carga suficiente para modificar la realidad; por el contrario, se asume que la transformación de los contextos se puede construir tanto desde la reflexión que induce la teoría de la resistencia, como junto a una postura crítica de la formación por competencias que no elude el compromiso sociohistórico.
\end{abstract}

Palabras claves: Formación profesional; educación superior; formación por competencias; discapacidad; kinesiólogos y kinesiólogas/fisioterapeutas. 
doi: http://doi.org/10.15359/ree.24-1.24

URL: http://www.una.ac.cr/educare

CORREO: educare@una.cr

\begin{abstract}
The essay identifies the structural factors of governmental power faced by people with physical dysfunction (PPD), integrating into the analysis the traditional training that kinesiologists / physiotherapists receive. The purpose of this approach is to show that the subsidies of the systemic structures are captured by a logic of reproduction that is born from the same world health organization (WHO), arriving to establish contradictorily before the complexity that this reality represents, a subsidiary conduct which holds the PPD responsible Faced with these events, a socio-critical approach is used to reveal that the structural constraints of power can only manage the insufficient existing subsidies. And that, in turn, this reality must be transformed into a prudent problematic nucleus to be incorporated into the formative processes of the curricular matrices of the physiotherapists who interact with property and frequently in these contexts. The document shows the socio-demographic scenario and the description of the status quo in order to interpret that with the theory of social reproduction or with the processes of official curricular innovation a sufficient burden is not achieved to modify reality, on the contrary, to assume that the transformation of the contexts can be built both from the reflection that induces the theory of resistance, and together with a critical position of training by competencies that does not elude sociohistorical commitment.
\end{abstract}

Keywords: Vocational training; higher education; competency-based training; disability, kinesiologists/physiotherapists.

\begin{abstract}
Resumo: $O$ ensaio identifica os fatores estruturais do poder governamental enfrentados por pessoas com disfunção física (PDF). Que e integrado ao treinamento tradicional que os profissionais de cinesiología/fisioterapia recebem. O objetivo dessa abordagem é mostrar que os subsídios das estruturas sistêmicas são capturados por uma lógica de reprodução nascida da Organização Mundial da Saúde (OMS) e se estabelecem, contraditoriamente, dada a complexidade que essa realidade representa, uma conduta subsidiária que responsabiliza as próprias PDF. Diante desses acontecimentos, a abordagem sócio-crítica é usada para revelar que as condições estruturais de poder só podem gerenciar os insuficientes subsídios existentes. Alem disso essa realidade deve ser transformada em um núcleo de problemas prioritários, a ser incorporado nos processos de formação das matrizes curriculares dos grupos de cinesiólogos / fisioterapeutas que frequentemente interagem com nesses contextos. $O$ documento mostra o cenário sócio demográfico e a descrição do status quo para interpretar que, com a teoria da reprodução social ou com os processos de inovação curricular oficial, não é alcancada uma carga suficiente para modificar a realidade; pelo contrário, supõe-se que a transformacao possa ser construida tanto a partir da reflexão induzida pela teoria da resistência, como para uma postura crítica do treinamento por competências que não contorne o compromisso sócio histórico.
\end{abstract}

Palavras-chave: Formação profissionalizante; ensino superior; formação baseada em competências; deficiência; cinesiologistas/fisioterapeutas.

\title{
Introducción
}

Como parámetro significativo de actuación profesional, en la praxis se constatan situaciones límites sobre la acción, cuando un ser humano inmóvil pierdela posibilidad de permanecer autónomo frente a una disfunción física (DF). Se sabe que tal contexto provoca dependencia (DorantesMendoza, Ávila-Funes, Mejía-Arango y Gutiérrez-Robledo, 2007), déficit para realizar actividades de la vida diaria, necesidad de asistencia para interactuar con el medio ambiente (Baltes y Wahl, 1990), apoyo para desenvolverse públicamente y donde definitivamente estas personas no pueden llevar Máximo Escobar-Cabello y Héctor Cárcamo-Váquez

Los artículos de la Revista Electrónica Educare del Centro de Investigación y Docencia en Educación de la Universidad Nacional, Costa Rica, se comparten bajo términos de la Licencia Creative Commons: Reconocimiento, No Comercial, Sin Obra Derivada 3.0 Costa Rica. Las autorizaciones adicionales a las aquí delimitadas se pueden obtener en el correo: educare@una.cr 
una vida libre de manera plena (OMS, 2001). En estas condiciones, enfrentar situaciones cotidianas, pero complejas, se transforma en un desafío hacia las normativas establecidas. Sin embargo, y para no abducir en extremo el escenario, es ilustrativo considerar que la condición previamente descrita, formará parte de cualquier ser humano en el ocaso de su vida donde experimentará la DF como parte ineludible de un proceso de sufrimiento natural, pero que está muy alejado del protagonismo soteriológico de la víctima (Carbullanca, 2014), a no ser que experimente la muerte súbita.

En este sentido, la DF afecta la decisión como acto fundamental de la existencia, no obstante que, en la intención de un cuerpo carente de movilidad, permanece presente siempre la voluntad de ser libre (Castro, 2006). Así el sujeto kinesiólogo/fisioterapeuta inmerso en semejante realidad ineludible, interactúa en su ejercicio profesional para comprenderla y constatar que es posible, inclusive ahí, establecer márgenes de maniobra susceptibles de una interpretación contra la arbitrariedad cultural de lo estructuralmente dado por la biopolítica del contexto (Foucault, 1967). Sin embargo, de la forma como se entienda el fenómeno, será la explicación que opere por medio de la tradición, la reproducción o la transformación que determine la postura asumida.

De esta manera, el propósito del presente manuscrito está dirigido a contextualizar los obstáculos que limitan a la persona con disfunción física (PDF), dando una mirada sociocrítica al proceso formativo del sujeto kinesiólogo/fisioterapeuta para mejorar su capacidad de resolución de problemas con base en fundamentos que impulsen acciones a favor de la persona dependiente a costo inclusive de los poderes hegemónicos. Todo esto con el soporte de las teorías de producción-reproducción social y resistencia, que como herramientas sirven para develar, si es en la formación profesional superior donde se pueden albergar o reforzar gran parte de las posibilidades de transformar esta situación.

\section{Desarrollo}

\section{El escenario de las personas con disfunción física}

Una mayor densidad de contenido al cuadro estructural, está dada por la Organización Mundial de Salud (OMS) en su política de Rehabilitación con Base Comunitaria (RBC), al plantear que "no se deberían seguir imponiendo servicios de rehabilitación" (OMS, 2005, p. 4), lo cual refuerza y delata la impronta asistencialista que ha gobernado esta realidad y que en lo profundo expone el entramado del lastre hegemónico de la superestructura ideológica sanitaria. Uno más de estos aspectos del campo es la restricción administrativa formal para el proceder profesional, lo cual se observa muchas veces como una acción que se torna inadmisible, inviable por demarcaciones protocolares de la burocracia. A pesar de que la decisión, en su justa medida y en el alcance del propósito terapéutico, es tan evidente que hacen parecer un despropósito la exigencia legal de los mecanismos necesarios para que una persona subsidiaria pueda movilizarse con integridad y dignidad. 
doi: http://doi.org/10.15359/ree.24-1.24

URL: http://www.una.ac.cr/educare

CORREO: educare@una.cr

El poder instalado no está dispuesto a renunciar a lo que son sus medios de control, su autonomía técnica y la organización de su servicio. Complementariamente, la persona dependiente, la inmóvil o la postrada con dificultad podrían rehusarse a esa tradición jerárquica. El conflicto surge porque aceptar tácitamente el poder de la autoridad técnica científica, homogeneíza las necesidades de las PDF al punto de que se establece una norma por sobre la realidad. Una norma que cuenta con sus propias realidades, donde quien administra ese espacio interpreta sus acciones de mundo como verdades absolutas sobre esa realidad. Es la jerarquía de la regla que se reproduce a fin de cuentas, concretándose en una privación más para el ser humano que está con una DF, la cual en la medida que sedimenta el peso legal, impide la autonomía de la praxis profesional la que, por definición, siempre es específica para el sujeto (Monteiro y Norman, 2013).

No obstante lo complejo del contexto funcional ${ }^{1}$ en cuestión, no es su polaridad deliberativa, sino que las actuaciones que se presentan en reiteradas oportunidades, sin una resolución adecuada del conflicto. Básicamente porque ello depende de la mantención de un proceso reproductivo del poder hegemónico, del habitus del sistema sanitario. Al ejercer la arbitrariedad cultural en el manejo de la enfermedad a través de un plan estereotipado de salud. La crisis se experimenta como una ruptura de la costumbre cuando frente a un conflicto, el retrospectivo plan de salud ya no proporciona criterios que validen y autoricen las respuestas, es decir, no se pueden valer de las mismas referencias que han estructurado el servicio a la fecha. El ejercicio reproductor no puede seguir apelando a los modos estandarizados a través de los cuales en el pasado se habrían manejado los conflictos. Frente a la crisis, es necesaria la ruptura de las normas que distinguen lo verdadero de lo falso (Arendt, 2011), para comprender el significado de las contradicciones, disfunciones y tensiones que existen en los órdenes sociales.

\section{La formación inicial necesita una dosis de resistencia}

Ahora bien, si lo que se necesita es revertir un inadecuado ejercicio del poder, en este determinado campo que sostiene un habitus específico. Una alternativa válida sería, entonces, interpelar a los entes responsables que producen y reproducen tales complicaciones. Pero, ¿qué se hace con aquellos entes que pudieran ser distinguidos como responsables directos?, es decir, los reproductores garantes de la tradición formadora, más directamente los cuerpos docentes.

Impedidos de un modo de control más radical, habría que solicitarles a esos grupos formadores que, introspectivamente, revisaren las cadenas cognitivas, los esquemas operatorios

\footnotetext{
${ }^{1}$ Contexto funcional: Parte con la consideración de que en palabras de Maureira (2017) función, es la expresión de movimiento que posee una intención, un impulso o un instinto fundamental y el contexto de la función se expresa concretamente en las capacidades que posee el individuo para resolver los problemas críticos impuestos por el medio ambiente, movilizando recursos de tipo físico, cognitivo y social, que lo habilitan para enfrentar, en forma autónoma y efectiva, las demandas impuestas. Cuando la DF impide la función autónoma para interactuar, es el contexto el que se transforma para mantener a la persona como un ser humano funcional.
} 
con sus respectivas familias de situaciones que soportan las acciones profesionales e incorporaran la necesidad de mirar críticamente sus metodologías formativas. Así, en la medida en las que tales relaciones productivas permanecieran carentes del desarrollo de capacidades vinculantes con la realidad y, por tanto, en connivencia con sistematizaciones irreductibles de una lógica conservadora de la formación, ajenas a los dilemas éticos de dependientes, podríamos especular con propiedad que se está ante la inmovilidad del funcionamiento de un sistema ideológico representativo y dominante del Estado, no sin desatender en ese cuadro que: "la resistencia de las clases explotadas puede encontrar [allí] ... medios y ... [ocasiones] de expresarse" (Althusser, 1989. p. 191).

De hecho, las teorías sociales críticas conceden un espectro de acción y permiten la posibilidad de que las ideas viabilicen un espacio concreto de transformación del ser (Freire, 1970) efectivamente por medio de hendiduras de resistencia, que apunten a cuestionar las determinantes visibles del conflicto formativo, donde el personal docente ha de acceder a una política adecuada que se ocupe del desarrollo más que de la regulación. Tal disposición contra hegemónica nos conduce a un personal educador situado, que contribuye con otras personas al mejoramiento y transformación de las relaciones de poder socio-cultural y económicas, más humanas y justas en la sociedad, territorio o comunidad que lo referencia (Pinto y Osorio, 2014). Asumiendo con fuerza la idea de que aún en condiciones de absoluta adversidad, el producto modelado por un sistema que reproduce, no necesariamente es hegemónico, sino que presenta fisuras y contradicciones que pueden ser ventajosamente aprovechados por proyectos alternativos (Giroux, 1992).

Hasta ahora, en este particular escenario analizado, las disposiciones legales o estructurales, si bien delegan responsabilidades, impiden otorgar autoridad para derivar soluciones integrales que consideren, en primer orden, al único sujeto de atención: el subsidiario de los derechos. De ahí con la crítica consistente y con el apoyo de las teorías sociales que permiten interpretar este particular fenómeno, en primer lugar, se dé una mirada desde la cual una alternativa al cambio sería transformar la formación inicial según el perfil de las necesidades pedagógicas del contexto (Hidalgo, 2017), para desarrollar intervenciones especializadas que modifiquen las consecuencias determinadas también en el terreno particular de las PDF.

\section{La magnitud del problema de las PDF}

En el contexto epidemiológico nacional, los criterios que categorizan la magnitud de las PDF están dados por el condicionamiento que genera la dependencia, la inmovilidad y, la postración. Refieren Acevedo, Alhers, Henríquez y Aguilera (2014) la siguiente estimación:

El 24,1\% de las personas de 60 años o más que tienen algún nivel de dependencia, sea esta leve, moderada o severa (Calvo A., Tartakowski A., Maffei T., 2001). Según el Estudio Nacional de la Dependencia en las Personas Mayores del año 2010 (SENAMA, 2010), la limitación funcional severa incluye a individuos postrados que están confinados a su 
doi: http://doi.org/10.15359/ree.24-1.24

URL: http://www.una.ac.cr/educare

CORREO: educare@una.cr

cama, sujetos que presenten demencia, de cualquier grado con puntaje MMSE corto $<13$ puntos y puntaje test de actividades funcionales de Pffefer $>5$ puntos (Quiroga P., Albala C., Klaasen G., 2004), presencia de incapacidad para efectuar 1 Actividad Básica de la Vida Diaria ... (ABVD) (Katz S., et col., 1964) con la excepción de bañarse o incapacidad para efectuar 2 Actividades Instrumentales de la Vida Diaria ... (AIVD) (Lawton M., Brody E., 1969). Las personas que cumplen con alguno de los 3 criterios mencionados, son consideradas como personas dependientes severas. (p. 22)

Si agregamos al análisis otras consecuencias de la disfunción motora provocada por las enfermedades no transmisibles, al menos el $60 \%$ de la población se encuentra en alguna categoría de déficit motor.

Complementariamente, según lo estudiado por la Organización Panamericana de Salud y Organización Mundial de la Salud (OPS y OMS, 2011), existen muy bajas tasas de profesionales en el Sistema Nacional de Servicios de Salud (0,1-0,2 x 10.000 habitantes) que se dediquen a este ámbito de disfunciones $y$, junto a los reducidos subsidios estatales, esta realidad condiciona la mejora de actividades atingentes a la recuperación de la funcionalidad de pacientes con DF. Al día de hoy un postrado en Chile tiene el derecho de recibir, con regularidad, solo una visita al semestre por parte del equipo de salud, lo cual es insuficiente en alto grado. A pesar de lo avanzado en las políticas, esta realidad administrativa mantiene una problemática social aún no resuelta. En contra parte, el mercado, si se considera la cantidad de escuelas que forman profesionales de la salud en todo el país, se han incrementado exponencialmente, lo cual configura un escenario que complejiza al estado respecto de la oferta terapéutica versus la demanda para esta población.

Si además, las personas tutoras de la población postrada no superan el 6,49 \% (Medina y Kaempfer, 2007) por una parte y, si la sociedad no reconoce esta problemática como un derecho básico por la otra, enfrentaremos situaciones similares a las publicadas por ComunidadMujer (2014):

[La magnitud del alcance], la gran mayoría de la población menor de 69 años piensa que la situación más deseable es el cuidado domiciliario (exclusivamente familiar en un 47.4 $\%$, y de contratación de personas para cuidado con participación familiar, en un 43.2 $\%$ ) [un antecedente que sorprende es que], a mayor nivel socioeconómico, menor es la frecuencia de opiniones a favor del cuidado domiciliario. (p. 9)

En estas circunstancias de no mediar una adecuada hermenéutica que transparente el sentido ético del análisis del problema, el derrotero que estas personas recorrerán en sus vidas dependientes, con una atención digna, y técnicamente acorde al desarrollo humano (Cruz y Hernández, 2006), terminará siendo una utopía más, de un país que ideológicamente se encuentra en vías de desarrollo, pero que en la práctica reduce la dimensión del problema. Expuesto el contexto, una interrogante lejana y perspicaz podría reverberar: ¿Qué hay detrás de esta situación? ... ideología, reproducción... ¿Qué impide dar una salida? 


\section{La salida formativa hegemónica}

Si buscamos una respuesta amplia y global a la crisis, no podemos desatendernos de la formación inicial de profesionales kinesiólogos/fisioterapeutas. Se puede especular que, en lo técnico, el proyecto altruista de la Organización de las Naciones Unidas para la Educación (UNESCO) sobre el actual perfil de las competencias universales, se centraba y apuntaba primordialmente a la consideración del desarrollo social y humano como una preocupación teórico epistemológico de primer orden en las formaciones disciplinares de profesionales. Es así como, para UNESCO resultaba absolutamente imprescindible la promoción de los conocimientos genéricos, transversales y los contenidos valóricos en la formación (UNESCO, 2005). Por otra parte, y más ideologizadamente, la Organización para la Cooperación y el Desarrollo Económico y el Banco Mundial (Organización para la Cooperación y el Desarrollo económicos [OCDE], 2013), en conjunto, promovieron la preparación de los recursos humanos, la mejora de la capacidad productiva y la calidad del trabajo. Estas últimas habilidades con referencia a la teoría del capital humano, que impulsa masivamente determinados estilos de reproducción en la realización de las funciones académicas tradicionales, para producir profesionales aptos al nuevo siglo.

Sin embargo, si se acude a las reducidas orientaciones formativas reguladas por el abierto mercado nacional, este indica con pragmatismo neoliberal que necesitamos profesionales para que se desempeñen en cualquier lugar del mundo, que trabajen en equipo, que sean bilingües angloparlantes, con alta flexibilidad para operar técnicamente y con buenas habilidades blandas (Comisión Nacional de Acreditación CNA, 2010). De verdad que se hace una quimera pensar que estos grupos profesionales devuelvan con generosidad la mano a su país, claro el Estado les ha cobrado cada segundo de educación recibida. Pareciera ser, entonces, que la empresa criolla, es la que se transforma en un referente que, por sobre problemáticas de re-significación contextual, impone a los programas académicos una pertinencia que atienda solo a los requerimientos de la oferta laboral o de proyectos rentables. Es decir, un grupo profesional universitario esterilizado de responsabilidad social, que debe rescatar su inversión económica a la brevedad ${ }^{2}$. Tales productos capitales serán los que deban generar una masa flexible de sujetos capacitados ampliamente para seguir disminuyendo los costos de producción, por sobre una solución de una evidente realidad particular y situada que ya dejó de ser solo una constatación estadística.

\section{El aporte de los modelos formativos de la renovación}

A estas alturas del relato, se puede aventurar una proyección sospechosa de la renovación curricular construida como una manifestación actualizada y obsecuente de la ideología

\footnotetext{
${ }^{2}$ En Chile la educación ha debido tener que extremar a tal punto su tolerancia conceptual que inclusive en opinión de un primer mandatario llegó a considerarse como: "Un bien de consumo".
} 
doi: http://doi.org/10.15359/ree.24-1.24

URL: http://www.una.ac.cr/educare

CORREO: educare@una.cr

dominante. Althusser (1989), en su alerta reproductiva nos indicaría que es necesario interrogar a la superestructura para develar, ¿cuáles son las señales de actualidad presentes en el bloque dominante, que están a la base del cambio en las relaciones sociales de producción?, o más específicamente... ¿cuáles de ellas estarían tensionando las relaciones humanas al punto que justificarían el cambio curricular? Probablemente la respuesta contemplaría: “Considerando que el funcionamiento de la ideología no tiene más historia que la historia de las sociedades de clases, "puro ensueño, ilusión...es decir nada" ..., nada que no sea reproducir materialmente la existencia de la práctica ritual defensora de la estructura.

Pero... insistamos en el punto, ¿cuál sería la fachada de la verdad que esconde la justificación de la actualización de los saberes en la innovación curricular?, esa verdad que interpretando a Foucault (1967) sería de la partida una condena por cierto al silencio de los discursos de los seres excluidos y, que agitaría los discursos de las verdades aceptables coludidas con los poderes institucionalizados.

Estas verdades que, en el caso del discurso de la formación profesional, pertenecen a los sujetos académicos "tradicionalistas" los cuales transmiten los códigos a pocos discípulos o discípulas, ... parafraseando a Los Prisioneros, "A otros dieron secretos que a mí no" (Lyon, 1986). Y que solo entre estos elegidos es posible la circulación y la transmisión del saber. Ese saber que mantiene el poder mediante distintos ritos académicos, con un lenguaje incomprensible e intimidatorio, cuyo propósito lo aleja conscientemente de la realidad cotidiana a la cual nos debemos. Pues quien tiene el poder, se mantiene y acaba imponiendo sus definiciones de realidad y de verdad (Ovejero y Pastor, 2001). Una verdad que convengamos ya contamos con que no necesariamente es una total realidad, no obstante, se prolonga en la realidad virtual mítica del servicio.

De ahí que se insista en lo sospechosa que resulta la cruzada que implementa la innovación en las metodologías de enseñanza, si es que, en su propósito no está la renovación de ese ejercicio de poder. En palabras de Ovejero y Pastor (2001), que replica Foucault: "La lucha por el saber es la lucha por el poder" (p. 102), de manera tal que, habiendo explorado los sustentos de la ideología y el poder del saber en el proceso de innovación curricular, se pueden escudriñar al menos dos interrogantes provocadoras:

1. ¿Estará en riesgo la producción de "capital humano adecuado", que el poder cree necesario modificar el saber reproductivo actual para hacer frente a las necesidades sociales?

2. Los nuevos requerimientos de los saberes, ¿hacen imprescindible mejorar el control ajustado de la dominación para mantener un poder productivo sobre los grupos neoformados? 
A efectos de volver al contexto, me apoyaré en un caso atingente, que puede ser utilizado como ejemplo para analizar los alcances que podría tener una enseñanza reguladora como técnica específica de la biopolítica (Saura y Luengo, 2015). Ubicada específicamente en el campo de la salud y la kinesiología, la relación entre la acción humana y la determinación estructural de las relaciones sociales, relaciones de poder, que están dadas preferentemente al centro del saber disciplinar. Al igual que otras semejantes e históricas relaciones de poder-saber en el contexto de salud, ellas fueron incorporadas por el prestigio y las influencias que a fines del siglo XIX formaron parte de otras más de las instrumentalizaciones del Estado moderno, para convertir un medio en un determinado fin.

El poder-saber construyó una disciplina de cuerpo al interior de las instituciones sanitarias que monopolizó un discurso reflejo del modo dominante de pensamiento y que las proyectó como un cuerpo interpretativo del mundo sobre las poblaciones sin salud, sin apoyo, es decir, sin el apoyo crítico de una formación crítica. De este modo, en el campo institucional se crearon las condiciones para definir unilateralmente lo que es normativo de lo que es el desvío de ello, esto es, la distinción entre lo normal y lo patológico, relaciones que confirman cómo el poder de la biomedicina es constantemente reproducido (Haraway, 1995). De modo que se abren espacios para la naturalización de las prácticas de sujeción analizadas desde el pensamiento foucaultiano.

Así, la delegación de autonomía que monopoliza conocimientos, prácticas, instituciones y su autoevaluación generó condiciones para convertir a los agentes de poder en jueces y parte, de lo que se creía que era lo moderno, separando arbitraria y epistemológicamente al objeto salud-enfermedad del sujeto función-disfunción. Con la clara justificación de que ambas combinaciones epidemiológicas representan una carga económica y social para el país, donde los abordajes se mantienen paradigmática y hegemónicamente a cargo del mismo e ineficiente poder, constituyendo ideológica y prácticamente un modelo que, a través de una relación de mando y control de las PDF, por años, replica una situación en la que no solo hay poder, sino violencia (Arendt, 2011); violencia simbólica (Bourdieau y Passseron, 1997).

De esta manera, la RBC declara de oficio que imponer asistencias en rehabilitación sin el consentimiento y la participación de las personas que los estén utilizando (OMS, 2005), tácitamente está reconociendo que tales consideraciones desde el prisma de la adopción de principios de mercado, con planteamientos regulatorios del ámbito privado y que son formas de actuación propias de los sectores empresariales, vienen a instalarse como un implante de una dinámica encubierta que desperfila y minimiza la trascendencia de una real reforma para mantener la regulación, la disciplina y la norma que, de suyo, no beneficia a las PDF (Saura y Luengo, 2015).

Tal escenario devela que sí, efectivamente, se necesita una razón consistente y resistente a la vez, para que estas relaciones de poder ameriten no solamente una transformación estética de los currículos, sino una mutación radical en la producción de otro modelo formativo para controlar la crisis de los vínculos de confianza en profesionales de la salud. Estos deben, sin 
doi: http://doi.org/10.15359/ree.24-1.24

URL: http://www.una.ac.cr/educare

CORREO: educare@una.cr

letargo, incorporar el análisis hermenéutico accediendo a la correcta tensión de las categorías propuestas por las teorías de la producción y la reproducción social, con especial atención en su dimensión simbólica (Bourdieau y Passeron, 1997), para el refuerzo de las explicaciones de los fenómenos en cuestión. Con ellas pasaremos a conocer uno más de los comportamientos hegemónicos del utilitarismo sistemático que impone a través de sus medios y lógicas la velada modernidad, un monopolio de la tradición formativa que pasó colada a constituirse en un "campo y habitus" de un ejercicio inamovible del quehacer diario en los centros educativos y sanitarios.

\section{Discusión}

La teoría de la resistencia permite interrogar la noción representada por los contextos funcionales de las PDF y ante las evidencias expuestas emerge el carácter de las relaciones sociales que subyacen y que permiten elaborar la contestación para desarrollar un discurso de transformación y emancipación. Sin embargo, en primer término es necesario precisar la magnitud o el nivel de reproducción que existe en el escenario analizado. Según Bourdieau y Wacquant (2012), campo es una red o una configuración de relaciones objetivas entre posiciones, dinámicas, contingentes que siguen una lógica que define un funcionamiento particular. Por tanto, se está en presencia de una institucionalidad que propone reglas, leyes, políticas, redes, servicios, instancias de formación para los agentes. Mientras que habitus corresponde a todo lo que, en este cuerpo estructurado, socializado, inscrito en el mundo moderno, se incorpore a las conductas inmanentes de este sector en particular. Estas configuraciones que básicamente mantienen el orden social a través de la interiorización de condiciones que determinan la posición objetiva de los agentes y su capacidad de intervención, son las expresiones materiales que determinan el carácter de transmisión y reproducción de conocimientos, cuya existencia y persistencia son necesarias tanto para el ejercicio de su función propia como de la inculcación de la arbitrariedad cultural de las que son productoras (Bourdieu y Passeron, 1997).

De este modo, se podría asumir que los dos supuestos de la innovación curricular tratados con anterioridad, tanto el de actualizar el saber reproductivo, como mejorar el control productivo, no corren riesgos ni presentan nuevos requerimientos de control que se desestabilicen por la imposición de un nuevo esquema educativo. Este debe adoptarse sin considerar los rasgos particulares de cada realidad, manteniendo la homogeneidad formativa de la tradición y tendiendo a reproducir irreflexivamente un mensaje unidireccional del poder hacia las instituciones encargadas de formar, solo como una expresión más de la política del gato pardo "cambiar todo para que no cambie nada".

Si los patrimonios inquisitivos del poder dimitieran los derechos para limitar, supervisar y dirigir el ejercicio de las ocupaciones y, en particular, la formación inicial legalizando la autonomía profesional, con derechos derivados de criterios habituales del propio saber y de 
las facultades a que solo los pares pudieran evaluar el propio trabajo y la toma de decisiones, fácilmente podríamos intuir que la restricción normativa indefectiblemente nos llevaría a tener una cadena de medios y fines que seguirían privilegiando la utilidad por sobre el significado. Si esto se salvaguarda así, y de no implementar un giro radical, corremos el riesgo de perpetuar la imposibilidad de establecer un proyecto diferente, que se construya para favorecer a los sujetos que esbozan su voluntad de ser más (Freire, 1970).

Para materializar la propuesta freiriana en un ser humano que sale de su opresión y se le concede la posibilidad ideal de que interactúe por intermedio de una comprensión crítica, con el atributo de una contribución formativa que incorpore una dosis de resistencia para mejorar la capacidad de resolver problemas. Es necesario que la estrategia de renovación profundice las actuaciones a través de las grietas que deja la arbitrariedad cultural, las que siempre están acompañadas de un espacio o fisura que arrastra el poder fáctico, abriendo un espacio para que las capacidades más importantes que han surgido de las teorías sociocríticas se expresen como un reconocimiento a que los mecanismos de reproducción pueden mutar hacia un sujeto emancipado.

En la tradición formativa de las decisiones se sabe que ellas derivan de probabilidades sobre eventos conocidos y, por ello, son universalmente válidos; no obstante, la validación particular, la validación de realidad, la validación de verdad... solo es en la individualidad de las PDF. Así la reiteración de que estos saberes-poder deben ser enseñados con apego a esa interacción académica, en tanto de por sí, representan un problema de análisis de contextualidad, dado que el supuesto indica que se hace imprescindible un acercamiento a la realidad social que demanda un capital humano adecuado, el cual comprende y asume que no solo se trata de resolver un problema a través de una técnica eficaz.

De este modo, si estamos en presencia de un grupo profesional con cierta base epistemológica, este naturalmente tiene dudas, pero no sobre la eficacia probabilística y universal de la técnica que maneja, sino que, de la verdad que a partir de la eficacia de las técnicas puede inferirse de las teorías que sustentan el origen del problema y, es en ese punto donde la estructura y por sobre todo la superestructura mantendrán, decididamente, sus privilegios que favorecen su actuar.

Con todo y aceptando que al capital humano adecuado le interesa transformar la realidad por medio de los supuestos de resistencia, recapitulemos en Freire (1970) para señalar que el ser humano siempre es un ser inacabado, y que al contrario de los propósitos de la educación bancaria y de su fuerza deshumanizadora, buscará la praxis: acción y reflexión de las personas sobre el mundo para transformarlo, por medio de una educación problematizadora que implica un desvelamiento de la realidad para que emerjan las conciencias de las que resulta la inserción crítica en la realidad para hacerla cada vez menos alienada. 
doi: http://doi.org/10.15359/ree.24-1.24

URL: http://www.una.ac.cr/educare

CORREO: educare@una.cr

Entonces, ya no bastará con la actividad de la contra cultura, que gran parte de su oposición a las etiquetas significados y valores de lo oculto y lo oficial hace evidente en los planes de estudio, sino que estará conformado por una ideología de la resistencia (Giroux, 1992). En esta, la contra lógica que conforma una cultura alterna subraya su propia realidad diferente y de forma más convincente propone su propia estrategia.

Por tanto, si la consolidación de la resolución de problemas que permite la renovación curricular en la formación inicial está en el marco de un margen de maniobras que requieren hacer efectivo el compromiso social, necesitamos recurrir a la teoría social crítica. Con especial referencia a la teoría de la resistencia, para escudriñar que la responsabilidad social puede contribuir a liberarnos del fino sistema hegemónico de control, para tributar a los sujetos pacientes que necesitan ser orientados y considerados en sus disfunciones (Figura 1). En tal caso, la teoría de la resistencia redefine las causas y el significado del comportamiento de oposición, al argumentar que tiene poco que ver con que una desadaptación sea aprendida y mucho más que ver con una indignación moral y política como fuente de cambio (Giroux, 1992).
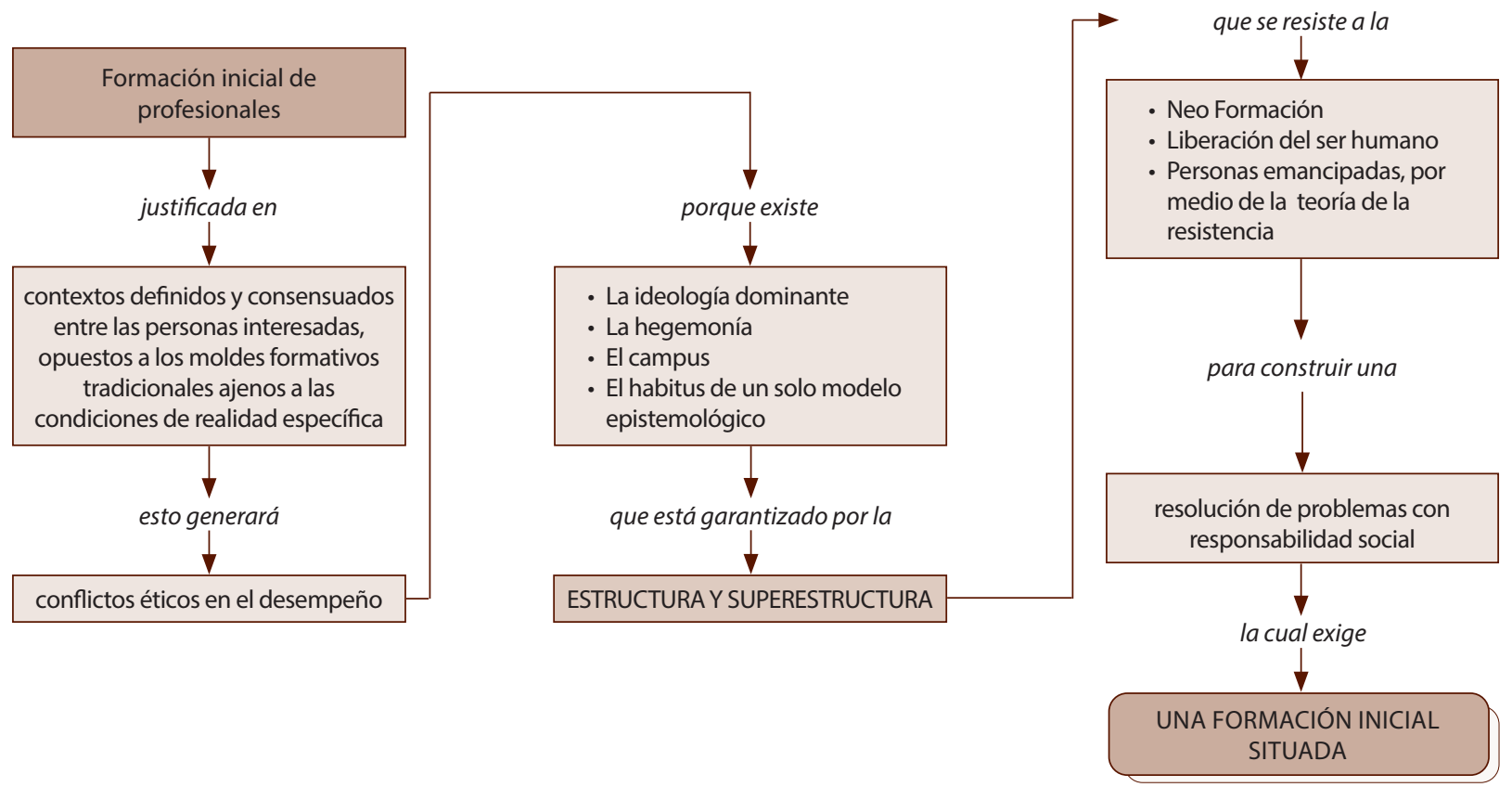

Figura 1. Elementos de contexto a considerar para dar una dosis de resistencia en los modelos de formación inicial.

Se expone la necesidad de modificar la formación inicial de profesionales considerando la contextualidad de los conflictos reales de la comunidad, los cuales requieren ser visualizados a través de las teorías sociales críticas utilizadas como herramientas al servicio de los análisis hermenéuticos que permiten identificar los alcances y el poder que ejercen sobre la mantención de modelos anacrónicos. Para la solución considera los notables aportes epistemológicos de autores sociocríticos que en base a la transformación y la superación del hombre, le otorgan un rol protagónico a la toma de decisiones situada en los diferentes centros de operación garantizando un accionar enunciado desde sus principios.

Nota: Elaboración propia. 
Finalmente, es en el contexto de las condicionantes éticas y morales intervinientes en los espacios de conflicto operacional, donde se hace necesario repensar el abordaje. Es ahí donde se focalizan variables que tensionan las decisiones críticas, las cuales están presentes en múltiples espacios de interacción como la negociación, la formación, la contradicción, para pasar a ser una fuente alternativa de acción competente.

\section{Conclusión}

La teoría de la resistencia sugiere que los educadores y las educadoras radicales deben desarrollar una relación más crítica que pragmática con sus estudiantes en la formación inicial. Esto quiere decir que cualquier forma viable de pedagogía radical debe analizar cómo se originan las relaciones de dominio, cómo se mantienen y cómo se relacionan con ellas los intervinientes. Esto implica ver más allá de las estructuras (Giroux, 1992).

Así, desvelar el punto crítico de la formación inicial, tomando en consideración la lógica inmutable que genera grupos profesionales inmunes a su pueblo sufriente, a sus problemas y denunciando a los tranquilos obedientes de la cultura tradicional que permanecen reproduciendo un modelo desgastado por la segregación y la inequidad social. Se impone un necesario rescate de la formación profesional mediante una dosis de resistencia que debe entenderse como la oportunidad de plasmar el desarrollo epistemológico disciplinar, con el objetivo de prepararse de manera competente para resolver problemas que presentan un compromiso sociohistórico (Gadamer, 2017). De esta manera, si estas dosis además están en la perspectiva de oponerse a la asistencialidad mercantilista que reciben las PDF en contextos donde la superestructura cubre con una política que refuerza la ideología del modelo neoliberal, podemos avanzar en los legítimos derechos de estos seres humanos, que están en la base del rol protector que la sociedad les ha encomendado celosamente a las instancias profesionales.

\section{Agradecimientos}

Al Dr. Omar Turra-Díaz, coordinador del Programa de Doctorado en Educación de la Universidad del Bío-Bío, por su inestimable juicio respecto de los productos de sus estudiantes, y al Prof. Óscar Bustos-Moyano por su asistencia técnica.

\section{Referencias}

Acevedo, J., Alhers, I., Henríquez, O. y Aguilera, H. (2014). Análisis de la discapacidad severa en Chile en pacientes atendidos en la atención primaria, año 2011-2012. Revista Chilena de Terapia Ocupacional, 14(1), 21-32. doi: https://doi.org/10.5354/0719-5346.2014.32385 
doi: http://doi.org/10.15359/ree.24-1.24

URL: http://www.una.ac.cr/educare

CORREO: educare@una.cr

Althusser, L. (1989). Ideología y aparatos ideológicos del Estado. En L. Althusser (Autor), La filosofía como arma de la revolución (18 ed., pp. 102-151). México: Siglo XXI.

Arendt, H. (2011). Entre o passado e o futuro (7a ed.). Saõ Paulo: Perspectiva.

Baltes, M. M. y Wahl, H. (1990). Dependencia en los ancianos. En L. L. Carstensen y B. A. Edelstein (Eds.), Gerontología clínica. Interención psicológica y social (pp. 40-57). Barcelona: Martínez Roca.

Bourdieau, P. y Passeron, J.-C. (1997). La reproducción. Elementos para una teoría del sistema de enseñanza. México: Editorial Laia. Recuperado de https://socioeducacion.files.wordpress. com/2011/05/bourdieu-pierre-la-reproduccion1.pdf

Bourdieau, P. y Wacquant, L. (2012). Una invitación a la sociología reflexiva (2a ed.). Argentina. Siglo XXI Editores. Recuperado de http://www.redmovimientos.mx/2016/wp-content/ uploads/2016/10/Bourdieu-P.-Wacquant-L.-2005.-Una-invitaci\%C3\%B3n-a-lasociolog\%C3\%ADa-reflexiva.-Editorial-Siglo-XXI.pdf

Carbullanca, C. (2014). Los mártires olvidados: Un estudio de los imaginarios del martirio en la fuente de los dichos. Veritas, 31, 135-160. doi: https://doi.org/10.4067/S0718$\underline{92732014000200007}$

Castro, R. (2006). Ética y libertad: La pars construens de la filosofía foucaltiana. Revista de Filosofía, 62, 117-138. doi: http://dx.doi.org/10.4067/S0718-43602006000100008

Comisión Nacional de Acreditación, CNA. (2010). Aseguramiento de calidad en la educación superior: Cuatro años de CNA-Chile (Memoria Institucional 2007-2010). Santiago: Autor. Recuperado de https://www.cnachile.cl/Biblioteca\%20Documentos\%20de\%20Interes/ MEMORIA.pdf

ComunidadMujer. (2014). Modelos de cuidado de personas adultas dependientes, cuidadoras y su participación en el mercado laboral (Informe final). Chile: Autor. Recuperado de http:// www.ministeriodesarrollosocial.gob.cl/btca/txtcompleto/com.muj-modeloscuidadosp. adultas.pdf

Cruz, I. y Hernández, J. (2006). Exclusión social y discapacidad. Colección textos de rehabilitación y desarrollo humano. Bogotá: Editorial Universidad del Rosario.

Dorantes-Mendoza, G., Ávila-Funes, J., Mejía-Arango, S. y Gutiérrez-Robledo, L. M. (2007). Factores asociados con la dependencia funcional en los adultos mayores: Un análisis secundario del Estudio Nacional sobre Salud y Envejecimiento en México 2001. Revista Panamericana de Salud Pública, 22(1). Recuperado de https://scielosp.org/pdf/rpsp/v22n1/a01v22n1.pdf 
Foucault, M. (1967). Historia de la locura en la época clásica I. México: Fondo de Cultura Económica. Recuperado de https://patriciolepe.files.wordpress.com/2007/06/foucaultmichel-historia-de-la-locura.pdf

Freire, P. (1970). Pedagogía del oprimido. Madrid. Siglo XXI Editores.

Gadamer, H.-G. (2017). Verdad y método. Salamanca: Ediciones Sígueme.

Giroux, H. (1992). Teoría y resistencia en educación. Una pedagogía para la oposición. México: Siglo XXI Editores.

Haraway, D. J. (1995). Ciencia, cyborgs y mujeres. La reivindicación de la naturaleza. Valencia: Cátedra.

Hidalgo, E. (2017). Hitos de la kinesiología: Su historia en Chile y otros temas valóricos relacionados. Santiago de Chile: Andros Impresores.

Lyon, A. (15 de setiembre de 1986). Prisioneros pateando piedras [Archivo de video]. Recuperado de https://www.youtube.com/watch?v=KVKOY7lalBw

Maureira, H. A. (2017). Síntesis de los principales elementos del modelo función-disfunción del movimiento humano. REEM, 4(1), 7-24. Recuperado de http://reem.cl/descargas/reem v4n1 a2.pdf

Medina, E. y Kaempffer, A. M. (2007). Medicina y otras carreras de la salud en Chile. Un análisis preliminar. Rev Médica de Chile, 135(10), 1346-1354. doi: http://dx.doi.org/10.4067/S0034$\underline{98872007001000018}$

Monteiro, S. y Norman, G. (2013). Diagnostic reasoning: Where we've been, where we're going. Teaching and Learning in Medicine, 25(S1), S26-S32. doi: https://doi.org/10.1080/1040133 $\underline{4.2013 .842911}$

Organización Mundial de la Salud (OMS). (2001). Clasificación internacional del funcionamiento, de la discapacidad y de la salud. Ministerio de Trabajo y Asuntos sociales. Secretaría General de Asuntos Sociales. Instituto de Migraciones y Servicios Sociales. Recuperado de http:// www.imserso.es/InterPresent2/groups/imserso/documents/binario/435cif.pdf

Organización Mundial de la Salud (OMS). (2005). RBC. Estrategia para la rehabilitación, la igualdad de oportunidades, la reducción de la pobreza y la integración social de las personas con discapacidad (Documento de posición conjunta). Suiza: Autor. Recuperado de https:// apps.who.int/iris/bitstream/handle/10665/43126/9243592386 spa.pdf;jsessionid=28CF 606FD61D7F9C5795007154E7231A?sequence $=1$ 
doi: http://doi.org/10.15359/ree.24-1.24

URL: http://www.una.ac.cr/educare

CORREO: educare@una.cr

Organización Panamericana de la Salud (OPS) y Organización Mundial de la Salud (OMS). (2011). Saluden Chile2010.Panorama delasituación desaludy delsistemadesaludenChile.Santiagom Chile: Autor. Recuperado de https://www.paho.org/chi/index.php?option=com docman\&view=download\&category slug=sistema-de-salud\&alias=101-la-salud-enchile-2010\&ltemid $=1145$

Organización para la Cooperación y el Desarrollo Económicos (OCDE). (2013). Panorama de la educación 2013. Indicadores de la OCDE. (Trad. Ministerio de Educación, Cultura y Deporte). España: Santillana. Recuperado de http://www.oecd.org/education/Panorama\%20de\%20 la\%20educacion\%202013.pdf

Ovejero, A. y Pastor, J. (2001). La dialéctica saber/poder en Michael Foucault: Un instrumento de reflexión crítica sobre la escuela. Aula Abierta, 77, 99-110. Recuperado de https://dialnet. unirioja.es/descarga/articulo/45498.pdf

Pinto, R. y Osorio, J. (2014). Pedagogía crítica para una educación pública y transformadora en América latina. Perú: Derrama Magisterial. Recuperado de http://www.comunidadvinculos. cl/wp-content/uploads/2016/07/Pedagogi\%CC\%81a-Cri\%CC\%81tica.pdf

Saura, G. y Luengo, J. (2015). Biopolítica y educación. Medición, estandarización, regularización poblacional. Teoría de la Educación, 27(2), 115-135. doi: http://dx.doi.org/10.14201/ teoredu2015272115135

UNESCO. (2005). Hacia las sociedades del conocimiento (Informe mundial de la Unesco). París: Autor. Recuperado de http://unescodoc.unesco.org/images/0014/001419/141908s.PDF 